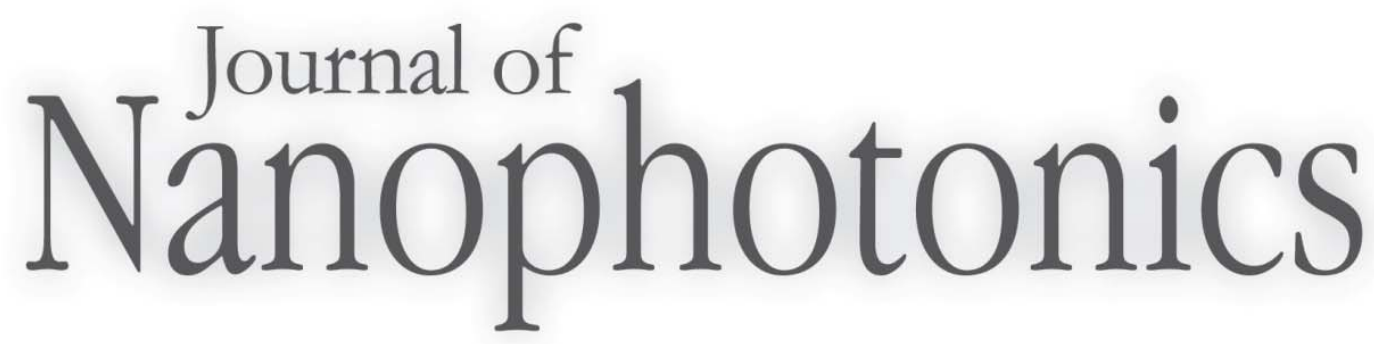

SPIEDigitalLibrary.org/jnp

\title{
Pole expansion of the Lorenz-Mie coefficients
}

Vadim A. Markel 


\title{
Pole expansion of the Lorenz-Mie coefficients
}

\author{
Vadim A. Markel \\ Departments of Radiology and Bioengineering, University of Pennsylvania, Philadelphia, PA \\ 19104 \\ vmarkel@mail.med.upenn.edu
}

\begin{abstract}
A spectral approach to the Lorenz-Mie problem was adopted to obtain a pole expansion of the Lorenz-Mie coefficients in the complex variable $z=4 \pi /\left(n^{2}-1\right)$, where $n^{2}$ is the dielectric permittivity of the scatterer. In the absence of magnetic properties (which is assumed), $n$ is the refractive index of the scatterer. It is shown that the Lorenz-Mie coefficients are meromorphic functions of $z$ with simple poles. The poles and the residues are functions of the size parameter $x=k a=2 \pi a / \lambda$ and of the order of the Lorenz-Mie coefficient, $l$, but are independent of the material properties. This leads to a numerically efficient representation of the Lorenz-Mie coefficients.
\end{abstract}

Keywords: Lorenz-Mie scattering, spectral theory, pole expansion

\section{INTRODUCTION}

The book Absorption and Scattering of Light by Small Particles by Bohren and Huffman [1] is regarded as classics and as an indispensable reference for many researchers working in the field of electromagnetic scattering. One of the book's lasting legacies is its excellent exposition of the Lorenz-Mie theory. In particular, an interesting remark which is important from the computational point of view can be found on p. 104 of the 1998 edition of the book. Here Bohren and Huffman discuss a figure in which the extinction efficiency, $Q_{\text {ext }}=\sigma_{\text {ext }} / 4 \pi a^{2}$ ( $\sigma_{\text {ext }}$ being the extinction cross section), of spherical water droplets of varying radius, $a$, is plotted as a function of the inverse wavelength, $1 / \lambda$, rather than as a function of the size parameter, $x=k a=2 \pi a / \lambda$. The latter way of representing extinction data was rather common at the time. Here is what Bohren and Huffman write (in the quote below, I use the notation " $n$ " for the refractive index instead of the original notation " $m$ "):

"This somewhat unconventional method of displaying extinction may cause some readers to reel in horror, particularly when it is noted that the curves in the figure ... show marked deviations from those more commonly encountered; extinction efficiencies are usually shown as functions of $x$ for a fixed refractive index $n$, a practice hallowed by tradition. Although the traditional method of displaying extinction is not necessarily incorrect, it is often misleading: $x$ and $n$ are mathematically independent variables but they may not be physically independent. This elementary fact is often lost sight of when $x$ is considered to be merely a dimensionless variable that is indifferent to whether it changes because of varying wavelength or radius. For if the wavelength varies, so is $n$ : no material substance has optical constants independent of wavelength except over a narrow range. ... The reason for the traditional method of displaying extinction has more to do with convenience than with fidelity to physical reality: it is relatively easy to calculate $Q_{\text {ext }}$ as a function of $x$ for fixed $n$. The curves shown in the figure ..., however, require considerably more effort than is usual: at each of the many wavelengths for which computations are done, the correct optical properties must be used."

Thus, the extinction efficiency can not be viewed as a function of a single scalar parameter $x$. This is because $x$ can change either due to a change in the particle radius or due to a change in the wavelength, and the latter occurrence is typically accompanied by a change of the refractive index, $n$, due to the effect of dispersion. The same can be said about the scattering and the absorption efficiencies. 
A natural question to ask is the following: Why has it to be the case that the computationally-intensive part of the Lorenz-Mie solution must be repeated every time a slight variation of parameters takes place, even when merely the sphere material is changed while the wavelength and the radius are kept constant? The difficulty can be understood by observing that the LorenzMie solution is not a spectral theory. This fact has been little appreciated, although, in principle, is known [2]. The vector spherical harmonics (VSHs) form an orthonormal basis and are solutions to the Maxwell's equations with constant coefficients in a spherical region and can, therefore, be used to construct a solution to the boundary value problem. However, the VSHs are not eigenfunctions of any "useful" linear operator. This statement will be made precise in Sec. 3 below. As a result, the Lorenz-Mie coefficients depend on both $x$ and $n x$ in a complicated way. Thus, when one of these parameters changes, the whole set of Lorenz-Mie coefficients must be recalculated.

There exists, however, a way to write the Lorenz-Mie coefficients in such a form that the most intensive part of the computation would depend only on the single parameter $x$. This can be achieved by utilizing a pole expansion of the Lorenz-Mie coefficients. As is well known, any meromorphic function of a complex variable $z, f(z)$, with simple finite poles $\alpha_{p}(p=1,2, \ldots)$ and residues $\beta_{p}$, can be written as

$$
f(z)=f(\infty)+\sum_{p} \frac{\beta_{p}}{z-\alpha_{p}}=f(0)+\sum_{p} \frac{\beta_{p} z}{\alpha_{p}\left(z-\alpha_{p}\right)} .
$$

For simplicity, I have assumed here that the function $f(z)$ is finite at $z=0$ and $z=\infty$, but the case when $f(z)$ has a pole at $z=0$ can easily be considered. In this paper, I will show that the Lorenz-Mie coefficients are meromorphic functions of the variable $z=4 \pi /\left(n^{2}-1\right)$, up to the overall factor $n^{-l}$ in the case of the internal field coefficients of the order $l$. Note that the Lorenz-Mie coefficients are finite for any values of $z$ such that $\operatorname{Im} z \leq 0$, which includes the points $z=0$ and $z=\infty$, so that the expansion (1) is applicable without restriction.

The variable $z$ defined above is known as the Bergman-Milton spectral parameter [3] and has been used primarily in the quasistatic limit. In a series of papers [4,5], I have shown that the same spectral parameter can be used even beyond the quasistatics. However, the development in these two references was rather formal. Here I will apply it specifically to the Lorenz-Mie problem.

The mathematical point of departure will be the scalar wave equation and I will consider scattering of scalar waves from a spherical object. This will yield expansions for the Lorenz-Mie coefficients $b_{l}, c_{l}$ which arise in the electromagnetic scattering theory and describe excitation of TE (transverse-electric) modes. The correspondence between the expansion coefficients for TE modes of the Maxwell's equations and the similar expansion coefficients which arise in the scalar scattering theory is quite complete. The electromagnetic theory, however, must include both TE and TM (transverse magnetic) modes; the latter have no analogy in the scalar theory. The Lorenz-Mie coefficients which correspond to the TM modes are $a_{l}$ and $d_{l}$; these coefficients are not considered explicitly in this paper, although the pole expansion for these coefficients should be obtainable in a similar manner without encountering a conceptual difficulty.

In what follows, I will obtain the pole expansion of the Lorenz-Mie coefficients $b_{l}$ and $c_{l}$ of the type (1) in which the coefficients $\alpha_{p}, \beta_{p}$ depend only on the size parameter $x$ and the order $l$. Once the poles and the residues are known as functions of $x$ and for all orders $l$ of interest, the Lorenz-Mie coefficients can be computed by simple summation. It should be noted that the functional form (1), apart from computational convenience, provides additional physical insight as it allows one to track and predict the appearance of spectral resonances.

The reminder of this paper is organized as follows. In Sec. 2, I will briefly review the mathematics of spectral methods and discuss some conceptual differences that arise when these methods are applied in the scattering theories of quantum mechanics and classical electrodynamics. 
In Sec. 3, I will derive the classical Lorenz-Mie solution for the scalar wave equation. In Sec. 4, I will show how a solution to the same problem can be obtained by a spectral method; the latter approach will naturally lead to a pole expansion of the Lorenz-Mie coefficients. In Sec. 5, I will discuss the numerical methods that can be used to compute certain new special functions which are related to the poles and the residues of the Lorenz-Mie coefficients. Numerical examples of such computations will be given. Finally, Sec. 6 contains a brief discussion.

\section{SPECTRAL METHODS IN PHYSICS}

From a mathematical point of view, the spectral methods are based on approximating the solutions to certain partial differential equations (PDEs) by truncated series of known, smooth functions [6]. The complete set of these functions must form a basis in an appropriate space. Of course, there are infinitely many choices for such bases. The basis which is typically used is the set of eigenfunctions of some relevant linear operator, e.g., of an operator that enters into the PDE. Generally, one seeks to write the PDE in the form

$$
(z-W)|\psi\rangle=|S\rangle
$$

where $z$ is a scalar spectral parameter, $W$ is a linear operator whose eigenfunctions are either known or can be found numerically, $|S\rangle$ is the known source, $|\psi\rangle$ is the unknown field. Assume for a moment that $W$ is Hermitian (self-adjoint). Then the spectral solution to (2) is

$$
|\psi\rangle=\sum_{\mu} \frac{\left|\psi_{\mu}\right\rangle\left\langle\psi_{\mu} \mid S\right\rangle}{z-w_{\mu}},
$$

where $\left|\psi_{\mu}\right\rangle$ and $w_{\mu}$ are the eigenfunctions and the eigenvalues of $W$ and $\sum_{\mu}$ may include integration over the continuous part of the spectrum.

The spectral variable $z$ and the operator $W$ in (2) are usually chosen in such a way that $z$ depends on a certain physical parameter which can be varied in an experiment while $W$ is independent of this parameter. To predict the results of different experiments in which $W$ is unchanged but $z$ varies, one needs to diagonalize $W$ only once. The solution is then obtained by using (3) for any value of $z$ by simple summation. Thus, the numerical complexity of the problem is greatly reduced as compared to the numerical complexity of inverting the operator $z-W$ in (2) for many different values of $z$.

For example, the scattering problem in quantum mechanics can be formulated as

$$
(E-H)\left|\psi_{\mathrm{s}}\right\rangle=U\left|\psi_{\mathrm{inc}}\right\rangle,
$$

where $E$ is the energy of incident particles, $H=-\left(\hbar^{2} / 2 m\right) \nabla^{2}+U$ is the Hamiltonian of the system, $U$ is the operator of potential energy and $\left|\psi_{\text {inc }}\right\rangle$ is the wave function that the particle would have in the case $U=0$. The total wave function is given by $|\psi\rangle=\left|\psi_{\text {inc }}\right\rangle+\left|\psi_{\mathrm{s}}\right\rangle$. The scattered component is obtained from (4) as

$$
\left|\psi_{\mathrm{s}}\right\rangle=\sum_{\mu} \frac{\left|\psi_{\mu}\right\rangle\left\langle\psi_{\mu}|U| \psi_{\mathrm{inc}}\right\rangle}{E-E_{\mu}}
$$

where $\left|\psi_{\mu}\right\rangle$ and $E_{\mu}$ are the eigenfunctions and eigenvalues of the Hamiltonian $H$ and integration over the continuous spectrum is assumed. We thus identify $z$ with the energy $E$ and $W$ with the Hamiltonian $H$.

Equation (5) is the spectral solution to the Schrodinger equation. It makes use of the fact that the interaction potential $U$ is physically and mathematically independent of the incident particle energy $E$. A scattering resonance would occur if $E$ could be tuned close to one of the discrete energy levels $E_{\mu}$. In an experiment, $E$ always belongs to the continuous spectrum 
and, therefore, it can not be equal to one of the discrete energy levels. However, scattering resonances at the so-called quasi-discrete states or at shallow bound states can be observed and are well known [7, § 134].

This example illustrates the broadly held view of the resonance as of the physical phenomenon which takes place when the driving frequency (or the incident particles energy) is close to one of the resonant values which usually belong to a discrete set. In this situation, the spectral variable $z$ is either the frequency or the energy. In the case of the electromagnetic scattering theory, this point of view may not be incorrect, but it is not very useful. Indeed, consider the electromagnetic counterpart of Eq. (4). In the frequency domain, the scattering equation reads

$$
\left[(\nabla \times \nabla \times)-k^{2}-4 \pi k^{2} \chi\right] \mathbf{E}_{s}=4 \pi k^{2} \chi \mathbf{E}_{\mathrm{inc}},
$$

where $k=\omega / c$ is the free space wave number, $\chi=(\epsilon-1) / 4 \pi, \epsilon$ is the complex, frequencydependent dielectric permittivity of the scatterer, and $\mathbf{E}$ is the electric field vector; the factor $4 \pi$ is introduced for historical reasons. If we now define $K=\nabla \times \nabla \times, U=-4 \pi k^{2} \chi, H=K+U$ and $E=k^{2}$, the equation becomes exactly of the form (4). The difficulty, as can be seen, arises from the fact that the interaction potential $U$ depends in this case on the frequency. There are two reasons for this dependence: the first reason is the prefactor $k^{2}$ and the second reason is the dependence of $\chi$ on $\omega$ due to the effect of dispersion. It is interesting to note that $\chi$ depends on the geometry and composition of the scattering object while all other quantities in Eq. (6) are medium-independent. The two types of frequency dependence mentioned above have somewhat different mathematical consequences. As will be seen below, the second type of dependence can be, in principle, removed by using a proper spectral parameter. The first type of dependence, however, is more fundamental and disappears only in the quasistatic limit, when the size of the scatterer is much smaller than the incident wavelength.

Thus, the use of the frequency (or of the quantity $k^{2}$ ) as the spectral parameter is problematic in the electromagnetic theory. At least, this provides no simplification similar to the one obtained in the quantum scattering theory. The question before us is then the following: Is there a better choice for the spectral parameter? In general, when $\epsilon(\mathbf{r})$ is an arbitrary function of position, no good answer to this question exists. However, in the case when $\epsilon(\mathbf{r})$ is piecewise constant and can take only two distinct values, a useful spectral parameter can be, in fact introduced.

For simplicity, assume that $\epsilon(\mathbf{r})$ can take the following two values:

$$
\epsilon(\mathbf{r})=\left\{\begin{array}{ll}
n^{2} \neq 1, & \text { if } \mathbf{r} \in V \\
1, & \text { otherwise }
\end{array},\right.
$$

where $V$ is the (possibly, disconnected) region occupied by the scattering material. The suitable spectral parameter is then $z=4 \pi /\left(n^{2}-1\right)$. This variable is known as the Bergman-Milton spectral parameter and the corresponding theory is the Bergman-Milton spectral theory [3]. The parameter $z$ defined above depends on the type of material and on the frequency due to the dispersion. It can be shown that the corresponding operator $W$ is independent of the material properties and defined only by the geometrical shape of the scatterer; it can still depend on the frequency due to the prefactor $k^{2}$, as discussed above. In the quasistatic limit, this dependence disappears and $W$ is completely defined by the scatterer shape, $V$. The explicit form of the operator $W$ will be given below in Sec. 4.

The Bergman-Milton theory has been used in many different settings, typically, within the quasistatics. For example, it was applied to the numerical analysis of multiple scattering and absorption by small aggregated spheres [8-11], to to compute the Casimir force between a sphere and a half-space [12], and in the electromagnetic theory of composites [13,14]. Generalizations of the theory beyond quasistatics were rarely considered, firstly, because of the onset of the 
frequency dependence of $W$, and, secondly, because $W$ ceases to be Hermitian and becomes complex symmetric at sufficiently high frequencies. I have used the theory of complex symmetric operators to generalize the Bergman-Milton approach to arbitrary frequencies in Refs. $[4,5]$. However, the dependence of $W$ on $\omega$ has rendered spectroscopic applications of this development (that is, computations involving multiple frequencies) problematic. Due to this reason, the generalized spectral theory of Refs. [4,5] has been applied to the situations in which the spatial properties of the field were of interest while the electromagnetic frequency was fixed, e.g., to study Anderson localization of monochromatic electromagnetic waves $[15,16]$.

However, in the special case of the Lorenz-Mie problem, it turns out that the operator $W$ defined according to Refs. [4,5] depends only on the single scalar parameter $x$. This fact is quite useful and will be exploited below.

\section{THE SCALAR MIE SOLUTION}

Consider the scalar wave equation

$$
\left[\nabla^{2}+\epsilon(\mathbf{r}) k^{2}\right] \psi(\mathbf{r})=0,
$$

where $\epsilon(\mathbf{r})$ is given by (7) where $V$ is region defined by $r \leq a, a$ being the sphere radius. The boundary conditions at the surface of discontinuity require that $\psi(\mathbf{r})$ be twice differentiable. The boundary condition at infinity is imposed on the scattered component of the field. Namely, we write $\psi=\psi_{\text {inc }}+\psi_{\mathrm{s}}$, where $\psi_{\text {inc }}$ satisfies Eq. (8) with $\epsilon=1$, and require that $\psi_{\mathrm{s}}$ vanish at infinity. Note, however, that there is no such requirement for the total field, $\psi$.

The solution to Eq. (8) with the boundary conditions specified is obtained in a straightforward manner. Let the incident field be a plane wave of the form

$$
\psi_{\mathrm{inc}}(\mathbf{r})=\exp \left(i \mathbf{k}_{\mathrm{inc}} \cdot \mathbf{r}\right)=4 \pi \sum_{l=0}^{\infty} \sum_{m=-l}^{l} i^{l} j_{l}(k r) Y_{l m}^{*}\left(\hat{\mathbf{k}}_{\mathrm{inc}}\right) Y_{l m}(\hat{\mathbf{r}}),
$$

where $\left|\mathbf{k}_{\text {inc }}\right|=k, \hat{\mathbf{r}}=\mathbf{r} / r$ is a unit vector, and the rest of notations are self-explanatory. We look for the solution in the form $\psi=\psi_{\text {in }}$ if $r \leq a$ and $\psi=\psi_{\text {inc }}+\psi_{\mathrm{s}}$ if $r>a$, and write $\psi_{\mathrm{s}}$ as

$$
\psi_{\mathbf{s}}(\mathbf{r})=\sum_{l=0}^{\infty} \sum_{m=-l}^{l} b_{l} h_{l}^{(1)}(k r) Y_{l m}^{*}\left(\hat{\mathbf{k}}_{\mathrm{inc}}\right) Y_{l m}(\hat{\mathbf{r}}) .
$$

Here the coefficients $b_{l}$ must be determined from the boundary conditions at the sphere surface. Analogously, the internal field is expanded as

$$
\psi_{\text {in }}(\mathbf{r})=\sum_{l=0}^{\infty} \sum_{m=-l}^{l} c_{l} j_{l}(n k r) Y_{l m}^{*}\left(\hat{\mathbf{k}}_{\mathrm{inc}}\right) Y_{l m}(\hat{\mathbf{r}}) .
$$

Here $n$ is the complex refractive index of the sphere. By applying the condition that $\psi(\mathbf{r})$ be twice differentiable at the sphere surface, we obtain the following expressions for the LorenzMie coefficients $b_{l}$ and $c_{l}$ :

$$
\begin{gathered}
b_{l}=4 \pi i^{l} \frac{j_{l}(n x) j_{l}^{\prime}(x)-n j_{l}(x) j_{l}^{\prime}(n x)}{n h_{l}^{(1)}(x) j_{l}^{\prime}(n x)-j_{l}(n x) h_{l}^{(1) \prime}(x)}, \\
c_{l}=\frac{1}{x^{2}} \frac{4 \pi i^{l-1}}{n h_{l}^{(1)}(x) j_{l}^{\prime}(n x)-j_{l}(n x) h_{l}^{(1) \prime}(x)},
\end{gathered}
$$


where prime denotes differentiation with respect to the argument in parentheses. Eqs. (10-12) give the complete solution to the scalar scattering problem. It should be emphasized that, up to the numerical factors of $-4 \pi i^{l}$ and $-4 \pi i^{l-1}$, respectively, the coefficients $b_{l}$ and $c_{l}$ are exactly the same as the Lorenz-Mie coefficients for the TE-polarized field modes which arise in the fully vectorial electromagnetic theory.

We now discuss what was meant in Sec. 1 by stating that the standard Lorenz-Mie solution $(10-12)$ is not a spectral solution. Let us write the partial wave of the order $(l, m)$ as

$$
\psi_{l m}(\mathbf{r})=f_{l}(r) Y_{l m}^{*}\left(\hat{\mathbf{k}}_{\mathrm{inc}}\right) Y_{l m}(\hat{\mathbf{r}}),
$$

where

$$
f_{l}(r)=\left\{\begin{array}{ll}
c_{l} j_{l}(n k r), & \text { if } r \leq a \\
4 \pi i^{l} j_{l}(k r)+b_{l} h_{l}^{(1)}(k r), & \text { if } r>a
\end{array} .\right.
$$

The functions $\psi_{l m}(\mathbf{r})$ are defined everywhere in space, satisfy Eq. (8), are twice differentiable and satisfy the boundary conditions at infinity. Therefore, these partial waves can be viewed, trivially, as eigenfunctions of the differential operator $\mathcal{L} \equiv \nabla^{2}+k^{2} \epsilon(\mathbf{r})$ with the single zero eigenvalue: $\mathcal{L} \psi_{l m}=0$. However, as was discussed in Sec. 2, construction of a spectral solution requires writing $\mathcal{L}$ as $\mathcal{L}=z-W$, where $z$ is a physical parameter that can be varied while $W$ is kept constant. But this has not been done in the case of the scalar Lorenz-Mie solution obtained above. Since $\psi_{l m}(\mathbf{r})$ are eigenfunctions of $\mathcal{L}$, they depend on all parameters of the problem. As a result, the coefficients $b_{l}$ and $c_{l}$ depend on both $k a$ and $n k a$ in a complicated way.

Note that the VSHs are often described as the "normal modes" (e.g., [1, $\S$ 4.3.2,4.3.3]). However, the normal modes are not equivalent to eigenfunctions, in the sense that they are not solutions to the frequency-domain Maxwell equations without an external source at any real frequency. However, VSHs can satisfy homogeneous equations for some complex frequencies which are known as the "natural frequencies" (e.g., [2]). The natural frequencies are singularities of the coefficients $b_{l}, c_{l}$, etc., viewed as functions of the complex frequency $\omega$. However, in the presence of dispersion, the normal modes are not eigenvalues of any easily definable linear operator and cannot be used to construct a spectral solution (at least, not without the use of approximations). The significance of natural frequencies is best revealed by studying the time evolution, e.g., after excitation by a short pulse. In particular, one can use the natural frequencies to compute radiative relaxation times [17].

\section{SPECTRAL APPROACH TO THE LORENZ-MIE PROBLEM}

We now formulate the spectral solution to the scalar Lorenz-Mie problem. We start by rewriting equivalently Eq. (8) in the integral form as

$$
\psi(\mathbf{r})=\psi_{\text {inc }}(\mathbf{r})+\frac{n^{2}-1}{4 \pi} \int_{V} G\left(\mathbf{r}, \mathbf{r}^{\prime}\right) \psi\left(\mathbf{r}^{\prime}\right) \mathrm{d}^{3} r^{\prime},
$$

where integration is extended over the volume of the sphere, $V$, and

$$
G\left(\mathbf{r}, \mathbf{r}^{\prime}\right)=k^{2} \frac{\exp \left(i k\left|\mathbf{r}-\mathbf{r}^{\prime}\right|\right)}{\left|\mathbf{r}-\mathbf{r}^{\prime}\right|}
$$

is the free space retarded Green's function for the Helmholtz equation which obeys

$$
\left(\nabla^{2}+k^{2}\right) G\left(\mathbf{r}, \mathbf{r}^{\prime}\right)=-4 \pi k^{2} \delta\left(\mathbf{r}-\mathbf{r}^{\prime}\right) .
$$

Note that if $\psi(\mathbf{r})$ is a solution to (15), it automatically satisfies all boundary conditions. Thus, the integral equation (15) is equivalent to the differential equation (8) plus the boundary conditions. 
Next, define the linear integral operator $W$ by

$$
W \psi(\mathbf{r}) \equiv \int_{V} G\left(\mathbf{r}, \mathbf{r}^{\prime}\right) \psi\left(\mathbf{r}^{\prime}\right) \mathrm{d}^{3} r^{\prime}
$$

and a spectral variable $z$ by

$$
z=\frac{4 \pi}{n^{2}-1}
$$

We can now re-write (15) in operator notations as

$$
(z-W)|\psi\rangle=z\left|\psi_{\text {inc }}\right\rangle .
$$

This equation has exactly the same form as (2), with $|S\rangle=z\left|\psi_{\text {inc }}\right\rangle$. The spectral variable $z$ and the operator $W$ possess the properties necessary for building a spectral theory: $z$ depends only on the material properties while $W$ is material-independent. In the case of a general scatterer, $W$ is defined by its geometrical shape and by the frequency $\omega$. However, in the case of the sphere, it is not difficult to see that $W$ is a function of the single parameter $x=k a$.

The operator $W$ is complex, symmetric and, hence, non-Hermitian (not self-adjoint). Eigenvalues of such operators are, generally, complex. An important fact is that $W$ is non-defective. An operator is called defective if at least one of its eigenvalues is degenerate and its geometric multiplicity is less than its algebraic multiplicity. Non-defectiveness of $W$ can be shown by considering subspaces spanned by eigenfunctions that correspond to each degenerate eigenvalue; proof is omitted but see the footnote below.

We now proceed with finding the eigenfunctions of $W$ and constructing a spectral solution to (20). Denote the eigenfunctions and eigenvalues of $W$ by $\psi_{\mu}(\mathbf{r})$ and $w_{\mu}$, respectively, so that

$$
W \psi_{\mu}(\mathbf{r})=\int_{V} G\left(\mathbf{r}, \mathbf{r}^{\prime}\right) \psi_{\mu}\left(\mathbf{r}^{\prime}\right) \mathrm{d}^{3} r^{\prime}=w_{\mu} \psi_{\mu}(\mathbf{r}) .
$$

Functions $\psi_{\mu}(\mathbf{r})$ are defined in the whole space, and Eq. (21) must also hold in the whole space, even though the integration in (21) is extended only over the volume of the sphere, $V$. With the use of (17), the eigenproblem (21) can be equivalently re-written in the differential form as

$$
-4 \pi k^{2} \Theta(\mathbf{r}) \psi_{\mu}(\mathbf{r})=w_{\mu}\left(\nabla^{2}+k^{2}\right) \psi_{\mu}(\mathbf{r}) .
$$

where $\Theta(\mathbf{r})=1$ if $\mathbf{r} \in V$ and $\Theta(\mathbf{r})=0$ otherwise (in a more general setting, $\Theta(\mathbf{r})$ can be referred to as the shape function $)$. The functions $\psi_{\mu}(\mathbf{r})$ must satisfy the same conditions as the scattered component of the field, $\psi_{\mathrm{s}}$, i.e., be twice differentiable and vanish at infinity. Equation (22) is somewhat similar to Eq. (8) but is not quite the same. To elucidate the differences, it is instructive to re-write (22) equivalently as

$$
\left[\nabla^{2}+k^{2}\left(1+4 \pi \frac{\Theta(\mathbf{r})}{w_{\mu}}\right)\right] \psi_{\mu}=0 .
$$

It can be seen that one of the differences is that Eq. (23) with the boundary conditions on $\psi_{\mu}(\mathbf{r})$ stated above has nontrivial solutions only for some special values of $w_{\mu}$ (which must necessarily be complex) while Eq. (8) has a nontrivial solution for arbitrary $\epsilon(\mathbf{r})$. This should become plausible if we recall that the total field $\psi(\mathbf{r})$ and the eigenfunctions $\psi_{\mu}(\mathbf{r})$ satisfy different boundary conditions at infinity.

Thus, just like the integral equation (21), the differential equation (22) is an eigenproblem, albeit of the generalized kind. In other words, it has the form $D \psi_{\mu}=w_{\mu} L \psi_{\mu}$, where $D$ and $L$ are two linear operators ( $D$ is diagonal). Even though generalized eigenproblems are usually more difficult to solve, the differential form (22) allows one to see that the eigenfunctions are 


$$
\psi_{l m p}(\mathbf{r})= \begin{cases}j_{l}\left[\eta_{l p}(x) k r\right] Y_{l m}(\hat{\mathbf{r}}), & r \leq a \\ A_{l p}(x) h_{l}^{(1)}(k r) Y_{l m}(\hat{\mathbf{r}}) & r>a\end{cases}
$$

where we have introduced the notation $\mu=(l, m, p), \eta_{l p}(x)$ is one of the complex roots (labeled by the index $p$ ) of the equation

$$
j_{l}(\eta x) h_{l}^{(1) \prime}(x)=\eta j_{l}^{\prime}(\eta x) h_{l}^{(1)}(x),
$$

which must be solved for $\eta$ for fixed $x$ and $l$, and

$$
A_{l p}(x)=j_{l}\left[\eta_{l p}(x) x\right]\left[h_{l}^{(1)}(x)\right]^{-1} .
$$

Note the recursion relation

$$
A_{l p}(x)=\eta_{l p}(x) A_{l+1, p}(x) .
$$

The eigenvalue corresponding to the triplet of indices $(l, m, p)$ is

$$
w_{l m p}(x)=4 \pi\left[\eta_{l p}^{2}(x)-1\right]^{-1} .
$$

Note the obvious degeneracy with respect to the projection of the angular momentum, $m$. Below, this index is omitted where appropriate *. Eqs. (25),(26) were obtained from the condition that $\psi_{l m p}(\mathbf{r})$ satisfies (22) and is twice differentiable. It can be verified directly, although by tedious integration, that $\psi_{l m p}(\mathbf{r})$ also satisfy the integral equation (21).

Equation (25) is parametrized by the order of the Bessel functions, $l$, and the size parameter, $x$. For each value of $l$ and $x$ it has many complex roots which we label by the index $p$. This gives rise to the special functions $\eta_{l p}(x)$ and $A_{l p}(x)$ which must be computed numerically by solving Eq. (25). The crucial fact, already mentioned above, is that both the eigenfunctions and the eigenvalues depend on the single scalar parameter $x=k a$ rather than on $k$ and $a$ separately. Further, the eigenfunctions and the eigenvalues are independent of the refractive index $n$ and, consequently, of the material properties of the scatterer. Not surprisingly, $\eta_{l p}(x)$ coincide with the values of the complex refractive index $n$ at which the Lorenz-Mie coefficients (12) have singularities (for a given value of $x$ ). On the other hand, in the standard approach to the LorenzMie problem, one is, typically, interested in singularities of the coefficients viewed as functions of the complex frequency, $\omega$, or in resonances observed when $\omega$ is scanned along the real axis.

Since the eigenfunctions $\psi_{l m p}(\mathbf{r})$ are not orthonormal in the usual sense (because $W$ is nonHermitian), we cannot use the usual orthogonality relations to construct a solution to (15). We, however, can exploit the property of quasi-orthogonality (see, for example, $[4,5])$ to construct a basis dual to $\psi_{l m p}(\mathbf{r})$. It is sufficient to find a dual basis in $L^{2}(V)$. Omitting intermediate steps, we state the final result. Namely, if

$$
\phi_{l m p}(\mathbf{r})=j_{l}^{*}\left[\eta_{l p}(x) k r\right] Y_{l m}(\hat{\mathbf{r}}), \quad r \leq a,
$$

then

$$
\int_{r \leq a} \phi_{l^{\prime} m^{\prime} p^{\prime}}^{*}(\mathbf{r}) \psi_{l m p}(\mathbf{r}) \mathrm{d}^{3} r=k^{-3} Z_{l p}(x) \delta_{l l^{\prime}} \delta_{m m^{\prime}} \delta_{p p^{\prime}}
$$

where

${ }^{*}$ The algebraic multiplicity of $w_{l p}$ is $2 l+1$ and is exactly equal to the geometric multiplicity, since all $2 l+1$ functions $Y_{l m}(\hat{\mathbf{r}})$ with $l$ fi xed and $m=-l, \ldots, l$ are linearly independent. 


$$
Z_{l p}(x)=\int_{0}^{x} t^{2} j_{l}^{2}\left[\eta_{l p}(x) t\right] \mathrm{d} t=\left.\frac{x^{2}}{2}\left[j_{l}^{2}(s)-(2 l+1) j_{l}(s) j_{l+1}(s)+s j_{l+1}^{2}(s)\right]\right|_{s=\eta_{l p}(x) x} .
$$

Thus, the functions $\phi_{l m p}(\mathbf{r})$ constitute a basis dual to the basis of the eigenfunctions $\psi_{l m p}(\mathbf{r})$ in $L^{2}(V)$. We now use this result to obtain a spectral solution to (15) (or (20)). First, let the total field inside the sphere be given by the following expansion: $\psi_{\text {in }}=\sum_{l m p} \beta_{l m p} \psi_{l m p}$. Substituting this directly into (15) and using (30), we find for the coefficients $\beta_{l m p}$ :

$$
\beta_{l m p}=\frac{k^{3} z\left\langle\phi_{l m p} \mid \psi_{\text {inc }}\right\rangle}{Z_{l p}(x)\left[z-w_{l p}(x)\right]} .
$$

The scalar product $\left\langle\phi_{l m p} \mid \psi_{\text {inc }}\right\rangle$ can be found with the use of formulas the (9) and (29):

$$
\begin{aligned}
\left\langle\phi_{l m p} \mid \psi_{\text {inc }}\right\rangle & =\int_{V} \phi_{l m p}^{*}(\mathbf{r}) \psi_{\text {inc }}(\mathbf{r}) \mathrm{d}^{3} r \\
& =4 \pi i^{l} Y_{l m}^{*}\left(\hat{\mathbf{k}}_{\mathrm{inc}}\right) \int_{0}^{a} r^{2} j_{l}\left[\eta_{l p}(x) k r\right] j_{l}(k r) \mathrm{d} r \\
& =i^{l-1} k^{-3} w_{l p}(x) A_{l p}(x) Y_{l m}^{*}\left(\hat{\mathbf{k}}_{\mathrm{inc}}\right) .
\end{aligned}
$$

We then arrive at the final result for the internal field:

$$
\psi_{\text {in }}(\mathbf{r})=\sum_{l m p} i^{l-1} \frac{w_{l p}(x) A_{l p}(x)}{Z_{l p}(x)} \frac{z}{z-w_{l p}(x)} j_{l}\left[\eta_{l p}(x) k r\right] Y_{l m}^{*}\left(\hat{\mathbf{k}}_{\mathrm{inc}}\right) Y_{l m}(\hat{\mathbf{r}}) .
$$

Analogous expression for the scattered field can be obtained in a straightforward manner. The result is

$$
\psi_{\mathbf{s}}(\mathbf{r})=\sum_{l m p} i^{l-1} \frac{\left[w_{l p}(x) A_{l p}(x)\right]^{2}}{Z_{l p}(x)} \frac{1}{z-w_{l p}(x)} h_{l}^{(1)}(k r) Y_{l m}^{*}\left(\hat{\mathbf{k}}_{\mathrm{inc}}\right) Y_{l m}(\hat{\mathbf{r}}) .
$$

Formulas (34),(35) give the spectral solution to the original problem. This solution has two remarkable properties: (i) the dielectric function of the scattering material enters only into the spectral variable $z=4 \pi /\left(n^{2}-1\right)$ and (ii) all coefficients (e.g., $\left.w_{l p}(x), A_{l p}(x), Z_{l p}(x)\right)$ are functions of the single parameter $x=k a$. We also note that summation over $m$ can be trivially performed with the use of the addition theorem for spherical functions.

By comparing (35) with the Lorenz-Mie solution (10),(12a), we find that

$$
b_{l}=i^{l-1} \sum_{p} \frac{\left[w_{l p}(x) A_{l p}(x)\right]^{2}}{Z_{l p}(x)} \frac{1}{z-w_{l p}(x)},
$$

This formula is simply the pole expansion of $b_{l}$. The key difference between this pole expansion and analogous formulas obtained in Ref. [2] is that here we view the Lorenz-Mie coefficient $b_{l}$ as functions of complex variable $z$, while in [2] they were viewed as functions of complex frequency $\omega$. The analogous expansion for the internal field coefficient $c_{l}$ reads

$$
c_{l}=i^{l-1} \sum_{p} \frac{w_{l p}(x) A_{l p}(x)}{Z_{l p}(x)}\left[\frac{\eta_{l p}(x)}{n}\right]^{l} \frac{z}{z-w_{l p}(x)},
$$

where $n=\sqrt{1+4 \pi / z}$ is the refractive index of the sphere and the square root branch is chosen so that $\operatorname{Im} n>0$. 
Another interesting formula can be obtained from (34) by considering the limit $z \rightarrow \infty$. In this limit, $\psi_{\text {in }}=\psi_{\text {inc }}$, from which we obtain

$$
j_{l}(y)=\frac{1}{4 \pi i} \sum_{p} \frac{w_{l p}(x) A_{l p}(x)}{Z_{l p}(x)} j_{l}\left[\eta_{l p}(x) y\right], \quad y \leq x .
$$

This equality holds in $L^{2}([0, x])$. Thus, $w_{l p}(x) A_{l p}(x) /\left[4 \pi i Z_{l p}(x)\right]$ are the expansion coefficients for the Bessel function $j_{l}(y)$ in the basis of $j_{l}\left[\eta_{l p}(x) y\right]$. By taking the limit $y \rightarrow 0$ and using (28), we find that

$$
\sum_{p} \frac{\eta_{l p}^{l}(x)}{\eta_{l p}^{2}(x)-1} \frac{A_{l p}(x)}{Z_{l p}(x)}=i, \quad \forall l, x .
$$

This sum rule may be used for verification of numerical results.

\section{SIMULATIONS}

I have investigated the feasibility of solving the transcendental equation (25). Two distinct numerical approaches to solving this equation have been tested. The first approach is based on polynomial approximation of transcendental functions and the second approach is iterative. These approaches are described in more detail below in Secs. 5.1, 5.2. The method of polynomial approximation was found to be quite stable, while a simple iterative method was not. However, only the simplest iterative scheme has been tested, and more sophisticated iterative approaches can still prove to be useful.

Numerical results for the functions $\eta_{l p}(x)$ and $w_{l p}(x)$ obtained by the method of polynomial approximation are shown in Fig. 1. The numerical solutions plotted in this figure satisfy Eq. (25) with very high precision (the relative error of the equation upon substitution of the numerical solutions is $10^{-14}$ or better). The figure shows parametric plots of the functions $\zeta_{l p}(x) \equiv$ $x \eta_{l p}(x)$ and $w_{l}(x)$ for $l=0, l=5$ and $l=10$.

\subsection{Method 1: Approximating (25) by an Algebraic Equation}

The first method is based on expressing the Bessel functions in (25) in terms of elementary functions, expanding all exponentials into truncated power series and approximating the transcendental equation (25) by a polynomial. This approach may be expected to yield accurate results only for roots of relatively small absolute value because, otherwise, the approximation of exponentials by polynomials is not accurate. This problem, however, can be bypassed by utilizing the periodicity of exponentials and by appropriately shifting the polynomials, as described below.

By using differentiation formulas, equation (25) can be written as

$$
j_{l}(\eta x) h_{l+1}^{(1)}(x)=\eta j_{l+1}(\eta x) h_{l}^{(1)}(x) .
$$

This must be viewed as an equation for $\eta$ parametrized by $l$ and $x$. If we make the substitution $\zeta=\eta x$, we also have an equation with respect to the variable $\zeta$ :

$$
x j_{l}(\zeta) h_{l+1}^{(1)}(x)=\zeta j_{l+1}(\zeta) h_{l}^{(1)}(x) .
$$

Further, we use the explicit expressions for the spherical Bessel functions in terms of elementary functions. After some algebraic manipulation, we obtain the following equation:

$$
P_{l+1}(-2 i x)\left[e^{i \zeta} P_{l}(-2 i \zeta)-e^{-i \zeta} P_{l}(2 i \zeta)\right]=P_{l}(-2 i x)\left[e^{i \zeta} P_{l+1}(-2 i \zeta)-e^{-i \zeta} P_{l+1}(2 i \zeta)\right]
$$



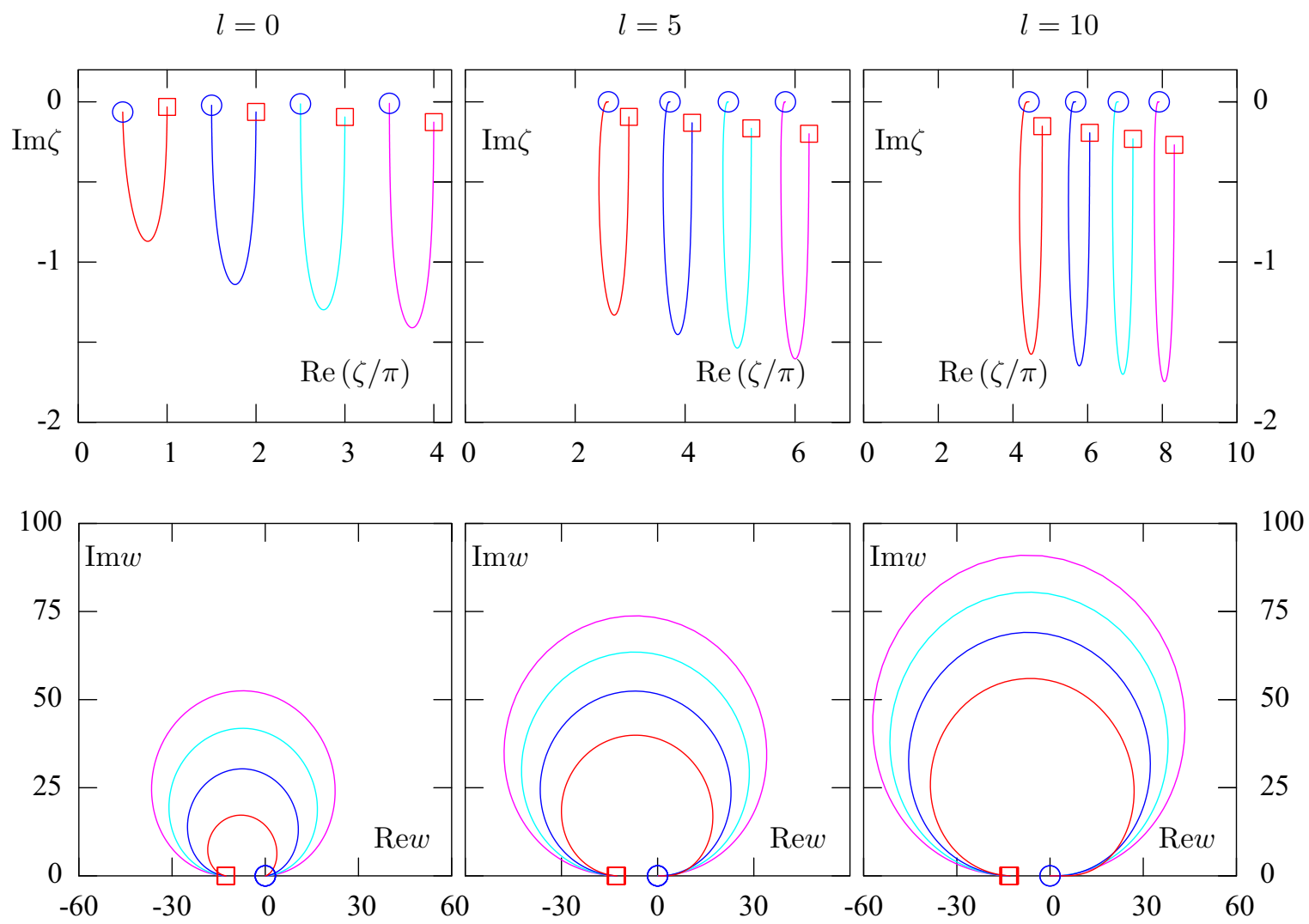

Fig. 1. Parametric plots of functions $\zeta_{l p}(x) \equiv x \eta_{l p}(x)$ (see Eqs. (40) and (41) below) and $w_{l p}(x)=4 \pi /\left[\eta_{l p}^{2}(x)-1\right]$ for $l=0$ (left column), $l=5$ (middle column) and $l=10$ (right column). Curves are plotted for $0.1 \leq x \leq 100$; the blue circles mark the points $x=0.1$ and red squares mark $x=100$. The red, dark blue, light blue, and purple curves correspond to different indices $p$. In the case $l=0$, these values are $p=1,2,3,4$, respectively. In the case $l=5$, there are no solutions for $p=1$, while the solutions for $p=2$ and $p=3$ form the same continuous curve (the red curve). In the case $l=10$, there are no solutions for $p=1,2,3$ while the solutions with $p=4,5,6$ form two separate continuous curves (the red and dark blue). 
where $P_{l}(x)$ are polynomials of the form

$$
P_{l}(x)=\sum_{k=0}^{l} \frac{(2 l-k) !}{k !(l-k) !} x^{k} .
$$

Next, we write $\zeta=\tau+p \pi$, where $-\pi / 2<\operatorname{Re} \tau \leq \pi / 2$ and $p$ is an integer. Obviously, any complex number $\zeta$ can be written in this form. Then we have

$$
e^{i \zeta}=(-1)^{p} e^{i \tau} \approx(-1)^{p} \sum_{k=0}^{k_{\max }} \frac{i^{k} \tau^{k}}{k !} .
$$

Here $k_{\max }$ is the maximum expansion order for the exponential. The above approximation is useful if the imaginary part of $\zeta$ (and, consequently, of $\tau$ ) is bounded. I have verified numerically that this is, indeed, the case. Using (44), we can re-write (42), approximately, as an algebraic equation of the order $N=l+k_{\max }+1$ in the variable $\tau$. This equation has $N$ complex roots. We are, however, seeking only the roots that satisfy $-\pi / 2<\operatorname{Re} \tau \leq \pi / 2$. We anticipate that there will be no more than one such root (this has been confirmed numerically for $l \leq 10$ ). We then repeat the numerical root-finding for different values of $p$, which will yield a family of roots $\tau_{l p}(x)$ (for fixed $l$ and $x$ ), and, correspondingly $\zeta_{l p}(x)=\tau_{l p}(x)+p \pi$, $\eta_{l p}(x)=\zeta_{l p}(x) / x$ and $w_{l m p}(x)=4 \pi /\left[\eta_{l p}^{2}(x)-1\right]$. Note that a numerically stable method for finding roots of a polynomial is diagonalization of the companion matrix. This method was used to compute the data points for Fig. 1.

One final remark is necessary. It turns out that the integer index $p$ defined above labels discontinuous segments of curves such as the ones shown in the figure. The curves can be relabeled so that the new index uniquely identifies a continuous curve. More details are given in the figure caption.

\subsection{Method 2: Iterative Root-Finding}

The iterative methods are based on evaluating the Bessel functions by methods whose numerical stability is well understood [18]. The simplest iterative method can be obtained by re-writing Eq. (41) as

$$
\zeta=\alpha_{l}(x) \frac{j_{l}(\zeta)}{j_{l+1}(\zeta)}
$$

where $\alpha_{l}(x)=x h_{l+1}^{(1)}(x) / h_{l}^{(1)}(x)$. Equation (45) can be iterated starting from an arbitrary initial guess. I have implemented this iteration and found that the map (45) is unstable, converging to a 2-cycle for sufficiently large iteration numbers.

A more sophisticated and, potentially, more stable approach is based on the Newton-Raphson method. Namely, fix $x$ and $l$ and define $F(\zeta)=\alpha_{l}(x) j_{l}(\zeta)-\beta_{l}(x) \zeta j_{l+1}(\zeta)$, where $\alpha_{l}(x)=$ $x h_{l+1}^{(1)}(x)$ and $\beta_{l}(x)=h_{l}^{(1)}(x)$. We then write the Newton-Raphson iteration as

$$
\zeta^{(n+1)}=\zeta^{(n)}-F\left[\zeta^{(n)}\right] / F^{\prime}\left[\zeta^{(n)}\right] .
$$

The derivative $F^{\prime}(\zeta)$ can be evaluated analytically by using the differentiation rules for Bessel functions. The natural choice of the initial guess is $\zeta^{(0)}=p \pi$, where $p$ is an integer. The different initial guesses can then generate a family of solutions. In order to avoid the possibility of $F^{\prime}\left[\zeta^{(0)}\right]$ approaching zero too closely, one can choose the initial guess with a small negative imaginary part, namely, $\zeta^{(0)}=p \pi-i \delta$. Solving Eq. (25) by the Newton-Raphson method has not been tested so far.

As a side note, the iterative methods can be used to improve the results obtained by the method of Sec. 5.1. In this case, the root obtained by the former method is used as the initial guess for an iterative method. 


\section{DISCUSSION}

Numerical computation of the special functions $\eta_{l p}(x)$ and the related functions $A_{l p}(x), Z_{l p}(x)$, $w_{l p}(x)$ will play the key role for the pole expansion of the Lorenz-Mie coefficients to become useful. It is logical to assume that, if these functions exist and are well defined, they can be computed with any given numerical precision. However, the choice of a numerical method is non-trivial. The functions $\eta_{l p}(x)$ must be computed by finding roots of a transcendental equation (25). The problem is complicated by the fact that the Bessel functions that enter into this equation themselves are known to be numerically unstable if evaluated "naively", e.g., by direct summation of polynomials. I plan to investigate this problem in the future by using a combination of approaches involving polynomial or rational Pade-type approximations and iterative methods.

Special attention should be given to convergence, stability and error estimates associated with the expansions (36), (37). The convergence of these expansions has not been investigated in this paper. It is known, however, that fast convergence is a general property of all "wellformulated" spectral solutions. Therefore, if the expansions (36), (37) are theoretically correct, it is reasonable to expect that convergence associated with the summation over the index $p$ should not pose a serious computational problem.

Finally, the spectral method presented in this paper might be generalizable to aggregated spheres (with the full account of interaction), photonic crystals composed of spherical or cylindrical inclusions, and to particles of other regular shapes.

\section{References}

[1] C. F. Bohren and D. R. Huffman, Absorption and Scattering of Light by Small Particles, Wiley, New York (1998).

[2] R. Fuchs and K. L. Kliewer, "Optical modes of vibration in an ionic crystal sphere," $J$. Opt. Soc. Am. 58, 319-330 (1968) [doi:10.1364/JOSA.58.000319].

[3] D. J. Bergman, "The dielectric constant of a composite material: A problem in classical physics," Phys. Rep. 43, 377-407 (1978) [doi:10.1016/0370-1573(78)90009-1].

[4] V. A. Markel, "Antisymmetrical optical states," J. Opt. Soc. Am. B 12(10), 1783-1791 (1995) [doi:10.1364/JOSAB.12.001783].

[5] V. A. Markel and E. Y. Poliakov, "Radiative relaxation time of quasi-normal optical modes in small dielectric particles," Phil. Mag. B 76, 895-909 (1997) [doi:080/01418639708243137].

[6] D. Gottlieb and S. A. Orszag, Numerical Analysis of Spectral Methods: Theory and Applications, SIAM, Philadelphia (1977).

[7] L. D. Landau, E. M. Lifshitz, and L. P. Pitaevskii, Quantum Mechanics (Non-relativistic Theory), Butterworth-Heinemann, Oxford (1977).

[8] R. Rojas and F. Claro, "Electromagnetic response of an array of particles: Normal-mode theory," Phys. Rev. B 34, 3730-3736 (1986) [doi:103/PhysRevB.34.3730].

[9] R. Fuchs and F. Claro, "Spectral representation for the polarizability of a collection of dielectric spheres," Phys. Rev. B 39, 3875-3878 (1989) [doi:103/PhysRevB.39.3875].

[10] F. Claro and R. Fuchs, "Collective surface modes in a fractal cluster of spheres," Phys. Rev. B 44, 4109-4116 (1991) [doi:103/PhysRevB.44.4109].

[11] V. A. Markel, V. N. Pustovit, S. V. Karpov, A. V. Obuschenko, V. S. Gerasimov, and I. L. Isaev, "Electromagnetic density of states and absorption of radiation by aggregates of nanospheres with multipole interactions," Phys. Rev. B 70, 054202 (2004) [doi:103/PhysRevB.70.054202].

[12] C. E. Roman-Velazquez, C. Noguez, C. Villarreal, and R. Esquivel-Sirvent, "Spectral representation of the nonretarded dispersive force between a sphere and a substrate," Phys. Rev. A 69, 042109 (2004) [doi:103/PhysRevA.69.042109]. 
[13] R. Fuchs, R. G. Barrera, and J. L. Carrillo, "Spectral representation of the electron energy loss in composite media," Phys. Rev. B 54, 12824-12834 (1996) [doi:103/PhysRevB.54.12824].

[14] M. I. Stockman, K. B. Kurlaev, and T. F. George, "Linear and nonlinear optical susceptibilities of maxwell garnett composites: Dipolar spectral theory," Phys. Rev. B 60, 1707117083 (1999) [doi:103/PhysRevB.60.17071].

[15] V. A. Markel, "Anderson localization of polar eigenmodes in random planar composites," J. Phys.: Condens. Matter 18, 11149-11165 (2006) [doi:088/0953-8984/18/49/009].

[16] V. A. Markel and A. K. Sarychev, "Propagation of surface plasmons in ordered and disordered chains of metal nanospheres," Phys. Rev. B 75, 085426 (2007) [doi:103/PhysRevB.75.085426].

[17] G. Y. Panasyuk, J. C. Schotland, and V. A. Markel, "Short-distance expansion for the electromagnetic half-space green's tensor: general results and an application to radiative lifetime computations," J. Phys. A 42, 275203 (2009) [doi:088/1751-8113/42/27/275203].

[18] S. Zhang and J. Jin, Computation of Special Functions, Wiley, New York (1996). 